Article

\title{
Robust Design of PC/ABS Filled with Nano Carbon Black for Electromagnetic Shielding Effectiveness and Surface Resistivity
}

\author{
Wipoo Sriseubsai ${ }^{1, * \mathbb{C}}$, Arsarin Tippayakraisorn ${ }^{1}$ and Jun Wei Lim ${ }^{2} \mathbb{C}$ \\ 1 Department of Industrial Engineering, Faculty of Engineering, King Mongkut's Institute of Technology \\ Ladkrabang, Bangkok 10520, Thailand; snoopynest_q@hotmail.com \\ 2 Department of Fundamental and Applied Sciences, HICoE-Centre for Biofuel and Biochemical Research, \\ Institute of Self-Sustainable Building, Universiti Teknologi PETRONAS, Perak 32610, Malaysia; \\ junwei.lim@utp.edu.my \\ * Correspondence: wipoo.sr@kmitl.ac.th; Tel.: +66-2-329-8339
}

Received: 31 March 2020; Accepted: 11 May 2020; Published: 21 May 2020

\begin{abstract}
This study focuses on the electromagnetic interference shielding effectiveness (EMI SE), dissipation of electrostatic discharge (ESD), and surface resistivity of polymer blends between polycarbonate (PC) and acrylonitrile-butadiene-styrene (ABS) filled with carbon black powder (CBp) and carbon black masterbatch $(\mathrm{CBm})$. The mixtures of PC/ABS/CB composites were prepared by the injection molding for the 4-mm thickness of the specimen. The D-optimal mixture design was applied in this experiment. The EMI SE was measured at the frequency of 800 and $900 \mathrm{MHz}$ with a network analyzer, MIL-STD-285. The result showed that the EMI SE was increased when the amount of filler increased. The surface resistivity of the composites was determined according to the ASTM D257. It was found that the surface resistivity of the plastic with no additives was $10^{12} \Omega /$ square. When the amount of fillers was added, the surface resistivity of plastic composites decreased to the range of $10^{6}-10^{11} \Omega$ /square, which was suitable for the application without the electrostatic discharge. The optimization of multi-response showed using high amounts of PC and CB was the best mixture of this research.
\end{abstract}

Keywords: PC/ABS; carbon black; electromagnetic shielding effectiveness; dissipation of electrostatic discharge; surface resistivity

\section{Introduction}

Nowadays, plastics, especially thermoplastics, are formed and used for many applications such as parts of automotive, electronic devices, and packaging. Some electronic devices generate and/or transmit electromagnetic waves that affect other devices, e.g., noise, an error operation, or the malfunction of electronic components [1]. An example is the capacitor in amplifiers that can generate electromagnetic waves that affect the quality of sound because of electromagnetic interference. Moreover, the electrostatic discharge transmitted from humans or tools may destroy some electronic parts. In order to prevent those problems, there were many researchers that have studied and developed electromagnetic interference shielding and dissipative material.

Generally, the material which has high performance for electromagnetic interference shielding effectiveness (EMI SE) is metal, due to high conductive properties. However, it has limitations such as weight, cost, processability, and corrosion [2]; then, plastic becomes the material of choice. There are many researchers who have developed and improved the EMI SE and dissipative plastic composites instead of metal, although normally, the plastic is electrically insulated and does not contribute to electromagnetic interference shielding. Plastic that is the matrix of the composite can connect the 
conductive filler. Plastic composites having conductive filler is one method to make an EMI shielding material. The filler can be aluminum flakes, steel fiber, or carbon fiber [3]. There are high demands of electrically conductive polymer, but it is not the same as plastic composites because of the poor processability. The conductive polymer does not require conductive filler in order to provide the shielding, so plastic composites with conductive filler are concerned and studied [3], with the increasing demand of customers for the reliability of electronic equipment [4-11]. Nanofillers that have been investigated by a number of researchers for EMI shielding were reviewed by Wanasinghe D. et al. [12]. It showed that nanocarbon black mixed with plastic made good shielding effectiveness, and the composite could have potential application in industry. However, the cost of the entire composite was high due to the nanoparticle production and additional material preparation process. Yangyong Wang and Xinli Jing studied EMI shielding by using polypyrrole (PPy) and polyaniline (PANI) [13], and the results showed the high performance of the shielding. Silver-palladium (AdPd) was coated to polyethylene terephthalate (PET) to be EMI shielding, and it was found that the shielding effectiveness depended on the conductive properties [14]. Quinton J. studied EMI and radio wave shielding with three additives, i.e., carbon, graphite, and carbon fiber, mixed with 2 types of polymer matrix, PA6.6 and polycarbonate (PC) [15]. The results showed that carbon black was more effective than other additives. Moreover, when using multiple additives, the shielding effectiveness was higher than using only one additive and related to the study of Pramanik et al. [16].

In addition, electrostatic discharge (ESD) is another problem when the insulation polymer has conductive property; it can cause the electrical equipment to be damaged. The resistance of the polymer is between conduction and insulation material, which is called static dissipative material. It has the surface resistivity between $10^{4}$ and $10^{11} \mathrm{ohm} / \mathrm{square}$, and it is used to make a product and prevent the electrostatic discharge [17].

PC is a high impact- and heat resistance, fair chemical resistance, and is transparent. ABS is a low-cost as well as flexible material. Both of them are widely used in many applications. Moreover, PC and ABS have been blended to get the advantages of both material properties for applications such as automotive, electronics and telecommunication, and medical devices. This research investigates PC/ABS mixed with carbon black powder (CBp) and carbon black masterbatch $(\mathrm{CBm})$ as electromagnetic interference shielding, the dissipation of electrostatic discharge (ESD) material, and surface resistivity. Carbon black powder is used as a filler for EMI, and it has been studied by many researchers for many applications, such as mixing with rubber to increase friction resistance and strain. Carbon black masterbatch is ready-mixed carbon black plastic. It can be added to compatible plastic during the forming of the product. It is easy to use compared with carbon black powder. The powder has to be compounded with a plastic matrix before forming, but the masterbatch can be added directly to the production process. However, the mixing ratio of the carbon black when using the masterbatch is more difficult to adjust than when using the powder grade. While a number of researchers have studied the effect of filler to EMI, this research studies the mixing ratio of each material, which is discussed and determined by the mixture design and statistical method to analyze and optimize the mixture of those materials.

\section{Materials and Methods}

Basically, plastic will have electromagnetic interference shielding effectiveness (EMI SE) property when it can act as the wave impedance and effect to the discontinuous electromagnetic field. When the electromagnetic waves attack the material, there are three mechanisms that polymeric material should have as shielding, such as reflection and absorption, so that little of the electromagnetic waves pass through that material [1] (as shown in Figure 1). This is defined as shielding effectiveness and can be determined by the following equation.

$$
S E=20 \log \frac{E_{1}}{E_{2}}=20 \log \frac{H_{1}}{H_{2}}
$$




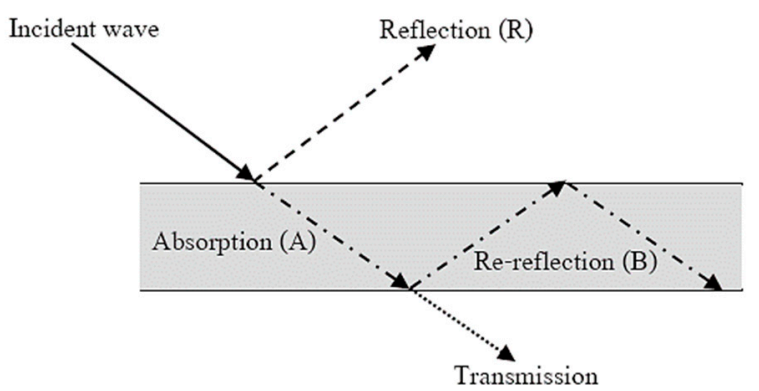

Figure 1. The mechanism of electromagnetic interference shielding effectiveness (EMI SE).

In Equation (1), $\mathrm{SE}$ is the shielding effectiveness, $\mathrm{dB} ; E_{1}, E_{2}$ are the amplitudes of the incident wave and transmitted wave $(\mathrm{V} / \mathrm{m})$, respectively; $H_{1}, H_{2}$ are incident and transmitted magnetic field strengths $(\mathrm{H} / \mathrm{m})$, respectively.

The development of composited plastic by conductive filler is one of the methods to get the electromagnetic interference shielding property. The mixtures of PC, ABS, and carbon black were prepared with the design of the experiment called a mixture design with the D-optimal method. This method is recommended when there are constraints in the proportions of the mixture components [18]. This research was limited to the mixture ratio by the viscosity of the mixture. When mixing with a high amount of carbon black, the viscosity of the composite material is increased. This would cause damage to the injection molding machine when the viscosity of the material is too high. Then, the mixture ratio of carbon black was limited by the mixture melt flow rate of $5 \mathrm{~g} / 10 \mathrm{~min}$, which was performed following the ASTM D1238. Then, the mixing of each composition by the mixture design with D-optimal was designed and is shown in Figure 2 and Table 1. The PC and ABS used in this research were commercial-grade 110 and PA 707, which were manufactured by CHIMEI. Two types of carbon black were used as the additive, i.e., 22-nm powder grade N220 manufactured by Thai Tokai Carbon Product and 26-nm commercial masterbatch PLASBLAK ${ }^{\circledR}$ UN2014 from COBOT.

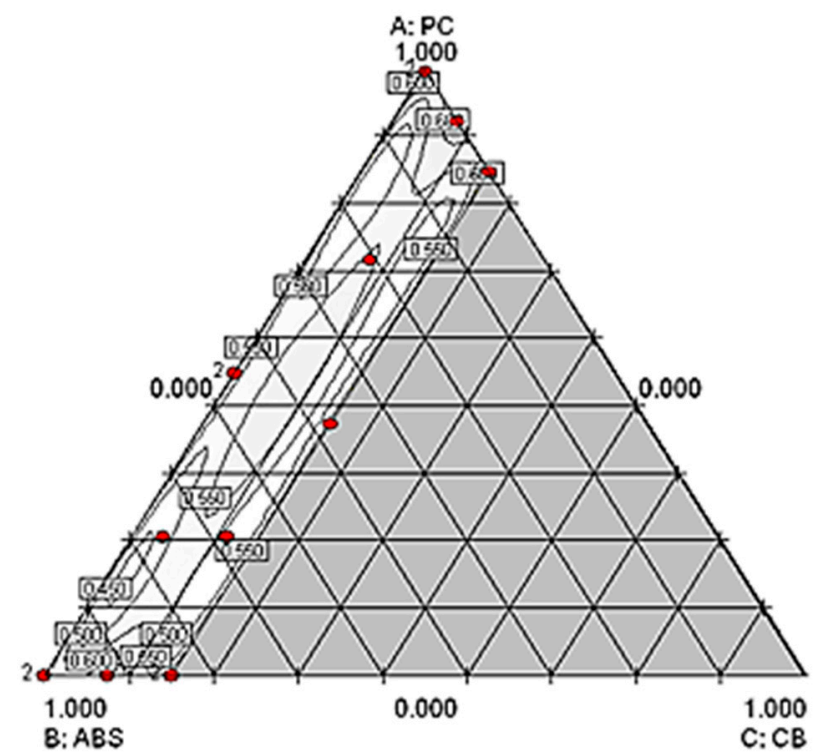

Figure 2. Mixture design. 
Table 1. Percentage of the composition of polycarbonate (PC)/ acrylonitrile-butadiene-styrene (ABS) and carbon black (CB).

\begin{tabular}{cccc}
\hline run & PC & ABS & CB (CBm, CBp) \\
\hline 1 & 0.00 & 0.83 & 0.17 \\
\hline 2 & 0.50 & 0.50 & 0.00 \\
\hline 3 & 0.00 & 0.83 & 0.17 \\
\hline 4 & 0.23 & 0.65 & 0.13 \\
\hline 5 & 0.23 & 0.73 & 0.04 \\
\hline 6 & 0.83 & 0.00 & 0.17 \\
\hline 7 & 0.42 & 0.42 & 0.17 \\
\hline 8 & 0.83 & 0.00 & 0.17 \\
\hline 9 & 0.69 & 0.23 & 0.08 \\
\hline 10 & 1.00 & 0.00 & 0.00 \\
\hline 11 & 0.00 & 0.92 & 0.08 \\
\hline 12 & 0.00 & 1.00 & 0.00 \\
\hline 13 & 1.00 & 0.00 & 0.00 \\
\hline 14 & 0.92 & 0.00 & 0.08 \\
\hline 15 & 0.00 & 1.00 & 0.00 \\
\hline 16 & 0.50 & 0.50 & 0.00 \\
\hline
\end{tabular}

All 16 combinations were mixed, and the plaque specimens performed with the dimension of $180 \times 100 \mathrm{~mm}$ and $4 \mathrm{~mm}$ thickness by an injection molding machine, Toshiba 80 Tons. The specimen was used to study electromagnetic interference shielding effectiveness by using the network analyzer MIL-STD-285, with the electromagnetic frequency of 800 and $900 \mathrm{MHz}$; the experimental setup is shown in Figure 3. The shielding effectiveness was determined by the following equation:

$$
\text { Shielding effectiveness }(\mathrm{SE})=\mathrm{P} 1-\mathrm{P} 2
$$

where $\mathrm{P}$ is the power level at Points 1 and 2 , respectively.



Figure 3. Source and receiver of the network analyzer. 
The dielectric constant was performed with the specimen dimension of $70 \times 100 \mathrm{~mm}$ and $4 \mathrm{~mm}$ thickness by using the Agilent $4263 \mathrm{~B}$ with $100 \mathrm{kHz}$ and $1000 \mathrm{mV}$. The parallel capacitance, $C_{p}$, was measured, and the dielectric constant was determined by the following equation:

$$
\varepsilon_{r}=\frac{t C_{p}}{A \varepsilon_{0}} \varepsilon=\varepsilon_{r} \varepsilon_{0}
$$

where

$\varepsilon$ is the dielectric constant $(\mathrm{F})$

$\varepsilon_{0}$ is $8.854 \times 10^{-12}(\mathrm{~F} / \mathrm{m})$

$\varepsilon_{r}$ is the relative dielectric constant

$C_{p}$ is the capacitance $(\mathrm{F})$

$A$ is the cross-section area (m)

$t$ is the thickness (m)

The surface resistivity was performed following the ASTM D257, as shown in Figure 4. The specimens were prepared as the plaque of $100 \times 100 \times 4 \mathrm{~mm}$. The surface resistance was measured, and the surface resistivity was determined by

$$
\sigma=\frac{R P}{g}
$$

where

$\sigma$ is the surface resistivity $(\Omega /$ square $)$

$R$ is the surface resistance $(\Omega)$

$P$ is the distance between electrodes $(\mathrm{cm})$

$g$ is the electrode circumference $(\mathrm{cm})$



Figure 4. Surface resistivity measurement following the ASTM D257.

\section{Results and Discussion}

According to the mixture design of the experiment, the electromagnetic interference shielding effectiveness of the mixture between PC/ABS and carbon black masterbatch and carbon black power for each testing frequency are shown in Figures 5 and 6, respectively. The results showed that when using a higher carbon black mixing ratio, the SE was increased by both testing frequencies because the additive is the conductive material, allowing the plastic composite to reflect and absorb the electromagnetic wave. The SE of the composite also showed a maximum value of about $9 \mathrm{~dB}$ at $800 \mathrm{MHz}$, and about $5 \mathrm{~dB}$ at $900 \mathrm{MHz}$ had been obtained for the mixing containing 17 wt \% carbon black. Moreover, the results showed that both plastic composites that used different carbon blacks had a slightly different effect on the SE because the size of the carbon black used was a small difference in size. 


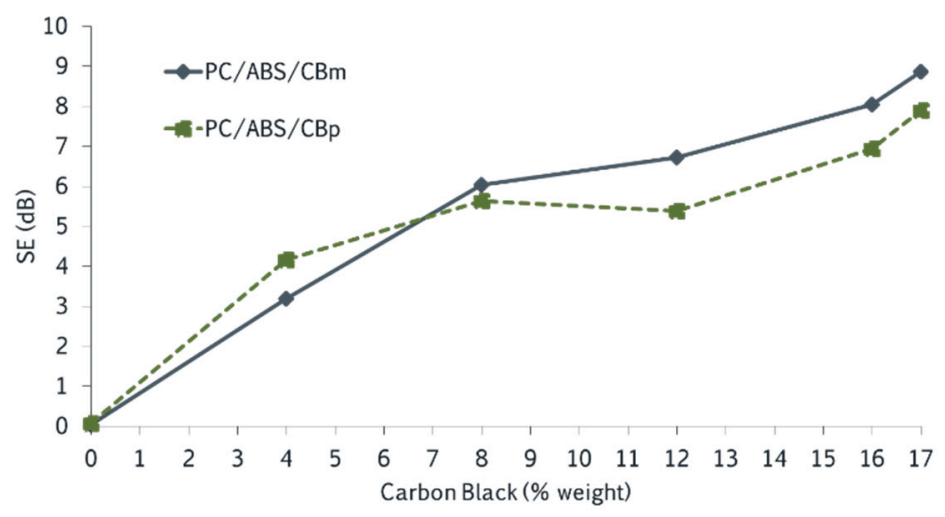

Figure 5. Shielding effectiveness (SE) at $800 \mathrm{MHz}$ with the carbon black.



Figure 6. SE at $900 \mathrm{MHz}$ with the carbon black.

The morphology studied of PC/ABS/CBp (0.42/0.42/0.16) and PC/ABS/CBm (0.69/0.23/0.08) conducted through SEM images is given in Figures 7 and 8, respectively, showing the proper distribution of carbon black within the plastic composite.



Figure 7. SEM image of the $16 \mathrm{wt} \%$ carbon black powder (CBp) in the PC/ABS. 


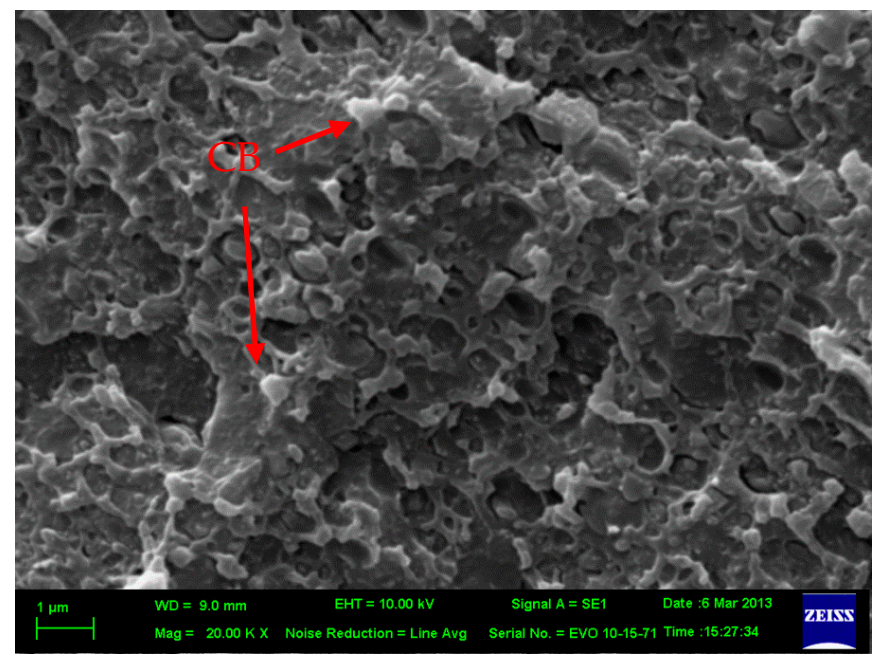

Figure 8. SEM image of the $8 \mathrm{wt} \%$ carbon black masterbatch $(\mathrm{CBm})$ in the PC/ABS.

The dielectric constant is the ability of a substance to store electric charge or electrostatic field energy [19]. When the dielectric is high, the material has low electrical insulation. The dielectric constant of mixing PC and ABS without carbon black in this research was between 3.04-3.34. After mixing $\mathrm{PC}$ and $\mathrm{ABS}$ with carbon black, the plastic composites were measured the dielectric constant by using the Agilent 4263B with $100 \mathrm{kHz}$ and $1000 \mathrm{mV}$. The results showed the dielectric constant was increased when the amount of carbon black in the mixture increased, as shown in Figure 9.



Figure 9. The relationship between dielectric constant and percentage of carbon black.

The maximum of the dielectric was about 25 when the plastic composite contained $17 \mathrm{wt} \%$ of carbon black, which was the upper limit of mixing carbon black for this research due to the high viscosity of the composite polymer. In contrast, the surface resistivity of the composite was the resistance to leakage current along the surface of an insulating material, which was decreased when the amount of carbon black filler increased. The surface resistivities were measured in both horizontal and vertical directions. The average surface resistivities of the composite are shown in Figure 10. The results show that when the composite contained $17 \mathrm{wt} \%$ of carbon black, the composite had the surface resistivity between $10^{7}-10^{8} \Omega$ /square, while the suitable surface resistivity for reducing the ESD of the plastic composite is between $10^{4}-10^{11} \Omega$ /square [17]. This is confirmation that carbon black, the conductive filler, is effective on the surface resistivity of the composite. The composite becomes a dissipative material when at least $5 \mathrm{wt} \%$ of carbon black is mixed. 




Figure 10. The relationship between surface resistivity and percentage of carbon black.

The EMI SE recorded data from the above experiments were used and analyzed by a statistical method. This method is a response surface methodology to determine the suitable regression model for the prediction of the EMI SE of the mixture. The statistical results, such as standard deviation, R-square, adjusted R-square, and PRESS, were analyzed for linear, quadratic, special cubic, and cubic models. When compared to those results, the adjusted R-square and R-square of the cubic model was higher than other models. Moreover, the standard deviation and PRESS of the cubic model were the lowest values when compared with other models. Then, the cubic model was selected for the prediction of the EMI SE of the mixture. The suitable regression model hypothesis was tested with ANOVA as well. The $p$-value and $p$-value of the lack of fit were statistically significant with $\alpha=0.05$. The model of those experiments is shown as the following:

At $800 \mathrm{MHz}$

Masterbatch:

$$
\begin{gathered}
\mathrm{SE}=-0.061 \mathrm{~A}+0.10 \mathrm{~B}+502.72 \mathrm{C}+0.14 \mathrm{AB}-819.99 \mathrm{AC}-816.79 \mathrm{BC}+811.86 \mathrm{ABC}+ \\
2.16 \mathrm{AB}(\mathrm{A}-\mathrm{B})+426.13 \mathrm{AC}(\mathrm{A}-\mathrm{C})+410.22 \mathrm{BC}(\mathrm{B}-\mathrm{C})
\end{gathered}
$$

Powder:

$$
\begin{aligned}
\mathrm{SE}=0.088 \mathrm{~A} & +0.081 \mathrm{~B}+4451.18 \mathrm{C}-0.20 \mathrm{AB}-7296.87 \mathrm{AC}-7286.17 \mathrm{BC}+6009.48 \mathrm{ABC} \\
& +2.72 \mathrm{AB}(\mathrm{A}-\mathrm{B})+3019.66 \mathrm{AC}(\mathrm{A}-\mathrm{C})+2999.24 \mathrm{BC}(\mathrm{B}-\mathrm{C})
\end{aligned}
$$

\section{At $900 \mathrm{MHz}$}

Masterbatch:

$$
\begin{gathered}
\mathrm{SE}=0.083 \mathrm{~A}+0.29 \mathrm{~B}+1483.98 \mathrm{C}-0.27 \mathrm{AB}-2402.47 \mathrm{AC}-2414.15 \mathrm{BC}+1961.35 \mathrm{ABC} \\
-4.65 \mathrm{AB}(\mathrm{A}-\mathrm{B})+988.02 \mathrm{AC}(\mathrm{A}-\mathrm{C})+996.78 \mathrm{BC}(\mathrm{B}-\mathrm{C})
\end{gathered}
$$

Powder:

$$
\begin{gathered}
\mathrm{SE}=0.035 \mathrm{~A}+0.17 \mathrm{~B}+1349.77 \mathrm{C}+0.75 \mathrm{AB}-2188.58 \mathrm{AC}-2148.86 \mathrm{BC}+1769.32 \mathrm{ABC}+ \\
2.12 \mathrm{AB}(\mathrm{A}-\mathrm{B})+918.63 \mathrm{AC}(\mathrm{A}-\mathrm{C})+855.09 \mathrm{BC}(\mathrm{B}-\mathrm{C})
\end{gathered}
$$

In Equations (5)-(8), $\mathrm{A}$ is $\mathrm{PC}, \mathrm{B}$ is $\mathrm{ABS}$, and $\mathrm{C}$ is carbon black, respectively. Those equations showed the independence and interaction of the factors. When considering the independent term, they show that carbon black $(C)$ is more effective to the SE than other factors. That was the reason why an increase in carbon black increased SE.

There were three data sets of the test: EMI SE, dielectric constant and surface resistivity. The dielectric constant and surface resistivity were related together and depended on each other. 
The optimization of the multi responses, EMI shielding effectiveness, and surface resistivity of each testing frequency was determined by using Design Expert software, while the level of PC/ABS/CB was the factor. The minimized parameters of the composite were determined by using the overlaid contour plot method and are shown in Figures 11-14. The results of SE and surface resistivity of the optimized $\mathrm{PC} / \mathrm{ABS} / \mathrm{CB}$ are also shown in Tables 2 and 3.



\section{Overlay Plot}

Figure 11. Overlay mapping @ $800 \mathrm{MHz}$ with carbon black masterbatch.

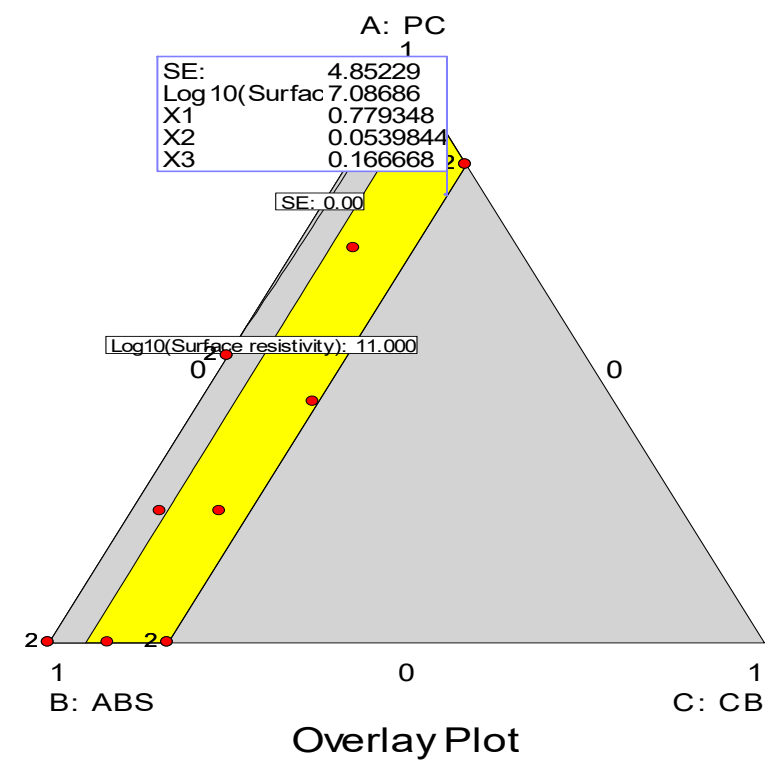

Figure 12. Overlay mapping @ $900 \mathrm{MHz}$ with carbon black masterbatch. 


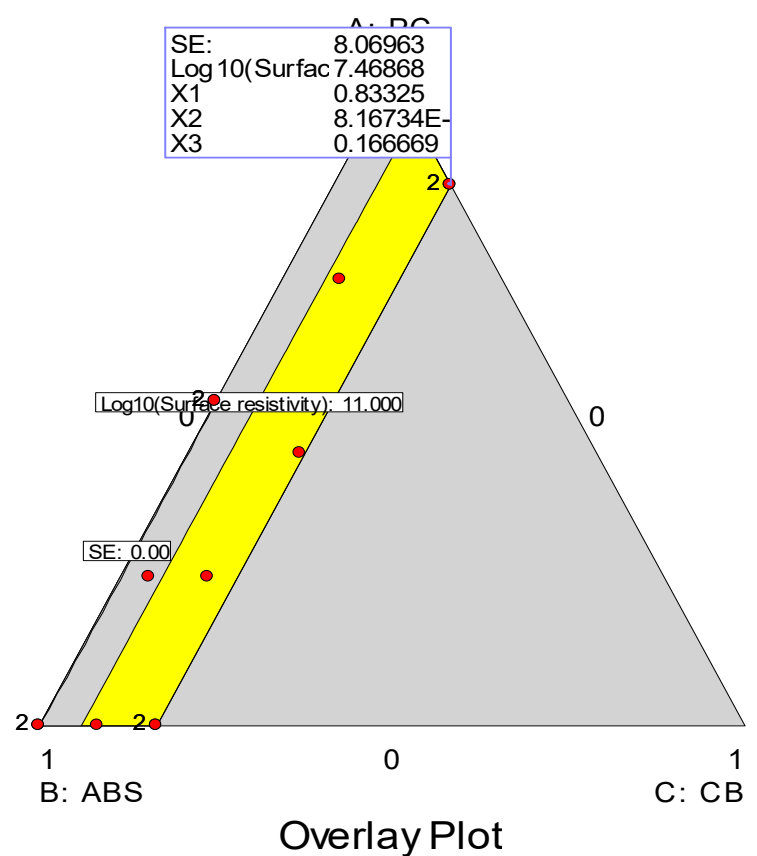

Figure 13. Overlay mapping @ $800 \mathrm{MHz}$ with carbon black particles.



Figure 14. Overlay mapping @ $900 \mathrm{MHz}$ with carbon black particles.

Table 2. Optimized mixing ratio among PC, ABS, and CBm.

\begin{tabular}{cccccc}
\hline Frequency & PC & ABS & CBm & SE & $\log _{\mathbf{1 0}}$ (Surface Resistivity) \\
\hline$@ 800$ & 0.83 & 0 & 0.17 & 9.31 & 7.09 \\
$@ 900$ & 0.78 & 0.05 & 0.17 & 4.86 & 7.08 \\
\hline
\end{tabular}

Table 3. Optimized mixing ratio among PC, ABS, and CBp.

\begin{tabular}{cccccc}
\hline Frequency & PC & ABS & CBp & SE & Log $_{10}$ (Surface Resistivity) \\
\hline$@ 800$ & 0.83 & 0 & 0.17 & 8.06 & 7.46 \\
$@ 900$ & 0.7 & 0.13 & 0.17 & 6.15 & 7.42 \\
\hline
\end{tabular}


The results showed that using a high amount of PC and CB optimized the mixture, which gave the high EMI shielding effectiveness for each testing frequency but gave the low surface resistivity that was about $10^{7} \Omega$ /square. It was also between the suitable range for reducing the ESD, $10^{4}-10^{11} \Omega /$ square [17]. At $800 \mathrm{MHz}$, the best composition of PC/ABS/CB was $0.83 / 0 / 0.17$ when using carbon black masterbatch or powder. At $900 \mathrm{MHz}$, the best composition of $\mathrm{PC} / \mathrm{ABS} / \mathrm{CBm}$ was $0.78 / 0.05 / 0.17$, but when using the powder carbon black, the best composition was $0.7 / 0.13 / 0.17$. While all the optimized compositions used a high percentage of PC, the EMI SE was high because the PC had a high polarity than ABS. The PC has polar side groups and regularity in the chain, while ABS has the polarity from the nitrile group. The polarity of the material may influence the shielding effectiveness of the composite as well.

\section{Conclusions}

The mixture of $\mathrm{PC} / \mathrm{ABS} / \mathrm{CB}$ that was studied in this research showed that $\mathrm{CB}$ influenced the EMI SE. The increasing $C B$ in the mixture affected the increasing electromagnetic interference shielding effectiveness and dielectric constant, but the surface resistivity was decreased. The design of experiments with the response surface method gave the suitable cubic regression model, which could predict those properties. The optimization of the mixture showed that a high amount of PC and CB gave better EMI SE. However, the $17 \mathrm{wt} \%$ of $\mathrm{CB}$ was the maximum level of this research due to the limitation of high viscosity. The electromagnetic field was reflected or absorbed by the composite due to the shielding property. The high polarity polymer was more significant than the low one. The size of carbon black from masterbatch and powder was not significant in this research. Both filler materials can be used to make the shielding polymer. However, the carbon black masterbatch is commercial-grade and easier to use than the powder. The powder grade is suitable when adjusting the mixture is often required. When $\mathrm{PC} / \mathrm{ABS}$ is required for shielding properties such as car audio components, it is recommended to add a high amount of $\mathrm{CB}$, and the ratio of PC should higher than ABS to get high EMI SE. However, this research suggests that the mechanical properties of the composite should be considered as an additional response because PC and ABS are blended to get the advantage of both material properties.

Author Contributions: Conceptualization, W.S.; methodology, W.S.; software, A.T.; validation, W.S. and A.T.; formal analysis, W.S.; investigation, W.S. and A.T.; resources, A.T.; data curation, A.T.; writing-original draft preparation, W.S. and A.T.; writing-review and editing, W.S. and J.W.L.; visualization, A.T.; supervision, W.S.; project administration, W.S. All authors have read and agreed to the published version of the manuscript.

Funding: This research was funded by King Mongkut's Institute of Technology, Ladkrabang, grant number CRT29-2561.

Acknowledgments: The authors wish to thank King Mongkut's Institute of Technology, Ladkrabang (Grant No. CRT29-2561), and the Faculty of Engineering, King Mongkut's Institute of Technology, Ladkrabang, where the experiments were performed.

Conflicts of Interest: The authors declare no conflict of interest.

\section{References}

1. Shuying, Y.; Lozano, K.; Lomeli, A.; Foltz, H.D.; Jones, R. Electromagnetic interference shielding effectiveness of carbon. Compos. Part A 2005, 36, 691-697.

2. Geetha, S.; Satheesh Kumar, K.K.; Rao, C.R.; Vijayan, M.; Trivedi, D.C. EMI Shilding: Method and Materials-A Review. J. Appl. Polym. Sci. 2009, 112, 2073-2086. [CrossRef]

3. Chung, D. Electromagnetic interference shielding effectiveness of carbon. Carbon 2001, 39, 279-285. [CrossRef]

4. Bjorklof, D. EMC Fundamentals Part Six: EMI filters and transient. Compliance Eng. 1998, 15, 10.

5. $\quad$ Brewer, R.; Fenical, G. Shielding: The hole problem. Eval. Eng. 1998, 37, S4-S10.

6. O'Shea, P. How to meet the shielding needs of a 500-MHz PC. Eval. Eng. 1998, 37, 40-46.

7. Ramasamy, S.R. Review of EMI shielding and suppression materials. In Proceedings of the International Conference Electromagnetic Interference and Compatibility, Piscataway, NJ, USA, 3-5 December 1997; pp. 459-466.

8. Geddes, B. Putting a Lid on EMI/RFI. Control (Chicago III) 1996, 9, 4. 
9. Hempelmann, S. Surface engineering for EMI compliance. Process and practical examples. Galvanotechnik $1997,88,418-424$.

10. Kimmel, W.D.; Gerke, D.D. Controlling EMI with cable shields. Med. Device Diagn. Ind. 1995, 17, 112-115.

11. Markstein, H.W. Effective shielding defeats EMI. Electron. Packag. Prod. 1995, 35, 4.

12. Wanasinghe, D.; Aslani, F.; Ma, G.; Habibi, D. Review of Polymer Composites with Diverse Nanofillers for Electromagnetic Interference Shielding. Nanomaterials 2020, 10, 541. [CrossRef] [PubMed]

13. Yangyong Wang and Xinli Jing, Intrinsically Conducting Polymers for Electromagnetic Interference Shielding. Polym. Adv. Technol. 2005, 16, 344-351. [CrossRef]

14. Lee, C.Y.; Lee, D.E.; Jeong, C.K.; Hong, Y.K.; Shim, J.H.; Joo, J.; Kim, M.S.; Lee, J.Y.; Jeong, S.H.; Byun, S.W.; et al. Electromagnetic Interference Shielding by Using Conductive Polypyrrole and Metal Compound Coated on Fabrics. Polym. Adv. Technol. 2002, 13, 577-583. [CrossRef]

15. Krueger, Q.J. Electromagnetic Interference and Radio Frequency Interference Shielding of Carbon-Filled Conductive Resins. Master's Thesis, Michigan Technological University, Houghton, MI, USA, 2002.

16. Pramanik, P.K.; Khastgir, D.; Saha, T.N. Electromagnatic Interference Shielding by Conductive Nitrile Rubber Composite Containing Carbon Filler. J. Elastomer Plast. 1991, 23, 345-361. [CrossRef]

17. Fundamentals of Electrostatic Discharge. Available online: www.https://incompliancemag.com/ (accessed on 1 March 2020).

18. Cornell, J.A. Experiments with Mixtures: Design, Models, and the Analysis of Mixture Data, 3rd ed.; John Wiley: Hoboken, NJ, USA, 2002; p. 400.

19. Plastics Design Library. Handbook of Plastics Joining A Practical Guide; Plastics Design Library: New York, NY, USA, 1997; p. 79.

(C) 2020 by the authors. Licensee MDPI, Basel, Switzerland. This article is an open access article distributed under the terms and conditions of the Creative Commons Attribution (CC BY) license (http://creativecommons.org/licenses/by/4.0/). 